[8] М. Г. К рей н, Об одной экстраполяционной проблеме А. М. Колмогорова, [8]

[9]

[9] P. Lov $y, A$ special probin of Gaussian random functions, Proceedings of the This Bit thematical Statistics and Probability, Vol. II. Probability Theory, Berkeley-Lo Angeles 1956.

[10] Б. Я. Л е ви н, Pacn ределение порней цельх фуньиий, Москва 1956.

[11] [12] Теория вероятностей п ее грименения IV (4) (1959), p. 465-471.

процессов, Теория вероятностей й еe тitributions, I, II, Paris 1950-1951.

[13] L. Schwartz, Théorie des distributions, I, II, Paris 1950
[14] E. C. Titchmarsh, The theory of functions, Oxford 1932.

[15] N. Wiener, Extrapolation, interpolation and smoothing of stationary time series, Cambridge - New York 1949.

MATHEMATICAL INSTITUTE OF THE POLISH ACADEMY OF SCIENCES INSTYTUT MATEMATYCZNY POLSKIEJ AKADEMII NAUK

Reçu par la Rédaction le 16.6.1961

\section{On modified Landau polynomials}

by

\section{J. RADECKI (Poznań)}

This paper contains some theorems on the approximation of continuous functions $f(t)$ in an infinite interval by means of polynomials

$$
P_{n}[f(t) ; x]=\frac{\int_{0}^{1} f\left(h_{n} t\right)\left[1-\left(t-\frac{x}{h_{n}}\right)^{2}\right]^{n} d t}{2 \int_{0}^{1}\left(1-t^{2}\right)^{n} d t},
$$

where $h_{n}>0$ and $\lim h_{n}=\infty$. This kind of polynomials were first introduced by Hsu $[1,2]$, who also showed their convergence in the case of $h_{n}=n^{\Theta}$ and $f(t)$ of certain classes of continuous functions. The results given in the present paper are more general.

THEOREM 1. If $x>0$, then $\lim _{n \rightarrow \infty} P_{n}(1 ; x)=1$ if and only if

$$
\lim _{n \rightarrow \infty} \frac{h_{n}}{\sqrt{n}}=0 \text {. }
$$

Proof. First we prove the sufficiency. Easy transformations give

$$
P_{n}(1 ; x)-1=\frac{\int_{x \sqrt{n} / h_{n}}^{\sqrt{n}}\left(1-\frac{u^{2}}{n}\right)^{n} d u+\int_{\left(1-x / h_{n}\right) \sqrt{n}}^{\sqrt{n}}\left(1-\frac{u^{2}}{n}\right)^{n} d u}{2 \int_{0}^{\sqrt{n}}\left(1-\frac{u^{2}}{n}\right)^{n} d u} .
$$

Since $\left(1-u^{2} / n\right)^{n} \leqslant e^{-u^{2}}$ for $|u| \leqslant \sqrt{n}$, we have

$$
0 \leqslant \int_{x \sqrt{n} / h_{n}}^{\sqrt{n}}\left(1-\frac{u^{2}}{n}\right)^{n} d u \leqslant \int_{x \sqrt{n} ; / h_{n}}^{\sqrt[1]{n}} e^{-u^{2}} d u,
$$




$$
0 \leqslant \int_{\left(1-x / h_{n}\right) \sqrt{n}}^{\sqrt{n}}\left(1-\frac{u^{2}}{n}\right)^{n} d u \leqslant \int_{\left(1-x / h_{n}\right) \sqrt{n}}^{\sqrt{n}} e^{-u^{2}} d u
$$

for sufficiently large $n$. Hence $P_{n}(1 ; x)-1 \rightarrow 0$ for

$$
\lim _{n \rightarrow \infty} \frac{\sqrt{n}}{h_{n}}=\infty \quad \text { and } \quad \lim _{n \rightarrow \infty} 2 \int_{0}^{\sqrt{n}}\left(1-\frac{u^{2}}{n}\right)^{n} d u=2 \int_{0}^{\infty} e^{-u^{2}} d u=\sqrt{\pi} .
$$

Now we prove the necessity. By (1), assuming $P_{n}(1 ; x) \rightarrow 1$, we have in particular $\int_{x \sqrt{n} / \hbar_{n}}^{\sqrt{n}}\left(1-u^{2} / n\right)^{n} d u \rightarrow 0$. Let us suppose the sequence $a_{n}=\sqrt{n} / h_{n}$ to have a finite point of accumulation $g \geqslant 0$. Then $a_{n_{k}} \rightarrow$ $\rightarrow g$ for an increasing sequence of positive integers $n_{k}$. Obviously

$\int_{x a_{n_{k}}}^{\sqrt[r]{n_{k}}}\left(1-u^{2} / n_{k}\right)^{n_{k}} d u \rightarrow 0$. Hence $\int_{x a_{m_{k}}}^{x\left(g_{+1}\right)}\left(1-t^{2} / n_{k}\right)^{n_{k}} d t \rightarrow 0$. Since the sequence

$\left(1-t^{2} / n\right)^{n}$ is convergent to $e^{-t 2}$ uniformly in every finite interval, the inequality $\left(1-t^{2} / n_{k}\right)^{n_{k}} \geqslant \frac{1}{2} e^{-t^{2}}$ is satisfied for every $t \in[0, x(g+1)]$ and sufficiently large $k$. Hence $\int_{x a_{n_{k}}}^{x(g+1)} e^{-t^{2}} d t \rightarrow 0$, for $0 \leqslant \frac{1}{2} \int_{x a_{n_{k}}}^{x(g+1)} e^{-t^{2}} d t \leqslant$ $\leqslant \int_{x a_{n_{k}}}^{x(g+1)}\left(1-t^{2} / n_{k}\right)^{n_{k}} d t$. On the other hand, $\int_{x a_{n_{k}}}^{x(g+1)} e^{-t^{2}} d t \rightarrow \int_{x g}^{x(g+1)} e^{-t^{2}} d t$ as $k \rightarrow \infty$. But this is impossible. Thus the sequence $a_{n}$ possesses no finite points of accumulation, i. e. $a_{n} \rightarrow \infty$.

Remark. It is easily seen that if $h_{n} / \sqrt{n} \rightarrow 0$, then the sequence $P_{n}(1 ; x)$ tends to 1 almost uniformly in $(0, \infty)$, i. e. uniformly in every subinterval of $(0, \infty)$.

THEOREM 2. If

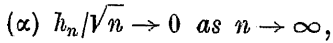

$(\beta)$ the sequence $\delta_{n}$ satisfies the conditions $\delta_{n}>0, \delta_{n} \rightarrow 0, \delta_{n} \sqrt{n} / h_{n} \rightarrow \infty$,

$(\gamma)$ the function $f(t)$ is measurable and bounded on each interval $[0, b]$,

( $\delta) f(t)$ is continuous at a fixed point $x>0$, then $P_{n}(f ; x) \rightarrow f(x)$ if and only if

(2) $\quad I_{n}(f ; x)=\frac{\sqrt{n}}{h_{n}} \int_{x+\delta_{n}}^{h_{n}} f(t)\left[1-\left(\frac{t-x}{h_{n}}\right)^{2}\right]^{n} d t \rightarrow 0 \quad$ as $\quad n \rightarrow \infty$.

Proof. The identity

$$
P_{n}(f ; x)-f(x)=P_{n}(f ; x)-f(x) P_{n}(1 ; x)+f(x)\left[P_{n}(1 ; x)-1\right]
$$

implies that $P_{n}(f ; x) \rightarrow f(x)$ as $n \rightarrow 0$ if and only if $P_{n}(f ; x)-$ $-f(x) P_{n}(1 ; x) \rightarrow 0$. Since

$$
\begin{aligned}
P_{n}(f ; x)-f(x) P_{n}(1 ; x) & =\frac{\int_{0}^{h_{n}}[f(t)-f(x)]\left[1-\left(\frac{t-x}{h_{n}}\right)^{2}\right]^{n} d t}{2 h_{n} \int_{0}^{1}\left(1-t^{2}\right)^{n} d t} \\
& =I_{n}(f ; x) \frac{1}{2 \sqrt{n} \int_{0}^{1}\left(1-t^{2}\right)^{n} d t}+a_{n}(x),
\end{aligned}
$$

where

(4) $\quad a_{n}(x)=$

$$
=\frac{\left(\int_{x-\delta_{n}}^{x+\delta_{n}}+\int_{0}^{x-\delta_{n}}\right)[f(u)-f(x)]\left[1-\left(\frac{u-x}{h_{n}}\right)^{2}\right]^{n} d u-f(x) \int_{x+\delta_{n}}^{h_{n}}\left[1-\left(\frac{u-x}{h_{n}}\right)^{2}\right]^{n} d u}{2 h_{n} \int_{0}^{1}\left(1-t^{2}\right)^{n} d t},
$$

it is sufficient to show that $\alpha_{n}(x) \rightarrow 0$, for $2 \sqrt{n} \int_{0}^{1}\left(1-t^{2}\right)^{n} d t \rightarrow \sqrt{\pi}$. However,

$$
\begin{aligned}
& \left|\alpha_{n}(x)\right| \leqslant \sup _{|u-x| \leqslant \delta_{m}}|f(u)-f(x)|+ \\
& \begin{array}{l}
+\frac{2 \sup _{0 \leqslant u \leqslant x}|f(u)| \int_{0}^{x-\delta_{n}}\left[1-\left(\frac{u-x}{h_{n}}\right)^{2}\right]^{n} d u+|f(x)| \int_{-+\delta_{n}}^{n_{n}}\left[1-\left(\frac{u-x}{h_{n}}\right)^{2}\right]^{n} d u}{2 h_{n} \int_{0}^{1}\left(1-t^{2}\right)^{n} d t} \\
\leqslant \sup _{|u-x| \leqslant \delta_{n}}|f(u)-f(x)|+\frac{2 \sup _{0 \leqslant u \leqslant x}|f(u)| \int_{\delta_{n} \sqrt{n} / h_{n}}^{x \sqrt{n} / h_{n}} e^{-t^{2}} d t+|f(x)| \int_{\delta_{n} \sqrt{n} / h_{n}}^{\left(h_{n}-x\right) v^{\prime} / h_{n}} e^{-t^{2}} d t}{2 \sqrt{n} \int_{0}^{1}\left(1-t^{2}\right)^{n} d t}
\end{array}
\end{aligned}
$$

for sufficiently large $n$. Since the function $f(t)$ is continuous at the point $x>0$ and the integral $\int_{0}^{\infty} e^{-t^{2}} d t$ is convergent, we obtain $\alpha_{n}(x) \rightarrow 0$.

THEOREM 3 . If

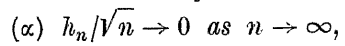

$(\beta)$ the sequence $\delta_{n}$ satisfies the conditions $\delta_{n}>0, \delta_{n} \rightarrow 0, \delta_{n} \sqrt{n} / h_{n} \rightarrow \infty$,

$(\gamma)$ the function $f(t)$ is continuous in the interval $[0, \infty)$, then $P_{n}(f ; x)$ is convergent to $f(x)$ almost uniformly in $(0, \infty)$ if and only if sequence (2) is convergent to zero almost uniformly in $(0, \infty)$. 
Proof. Applying identity (3), it is sufficient to prove that sequence (4) is convergent to zero almost uniformly in $(0, \infty)$. Let $x \in[a, b]$, where $a>0$. We have

$$
\begin{aligned}
&\left|\alpha_{n}(x)\right| \leqslant \sup _{\substack{|u-x| \leqslant \delta_{n} \\
x \in[a, b]}}|f(u)-f(x)|+ \\
&+\frac{2 \sup _{0 \leqslant \leqslant \leqslant b}|f(u)| \int_{\delta_{n}} \sqrt{n} / h_{n}}{b \sqrt{n} / h_{n}} e^{-t^{2}} d t+\sup _{0 \leqslant x \leqslant b}|f(x)| \int_{\delta_{n} \sqrt{n} / h_{n}}^{\left(h_{n}-a\right) \sqrt{n} / h_{n}} e^{-t^{2}} d t \\
& 2 \sqrt{n} \int_{0}^{1}\left(1-t^{2}\right)^{n} d t
\end{aligned}
$$

for sufficiently large $n$. By the uniform continuity of $f(t)$, the sequence $a_{n}(x)$ is convergent to zero uniformly in $[a, b]$. The interval $[a, b]$ being arbitrary, $a_{n}(x) \rightarrow 0$ almost uniformly in $(0, \infty)$.

\section{COROLLARY 1. If}

(a) $h_{n} / \sqrt{n} \rightarrow 0$ as $n \rightarrow \infty$,

( $\beta) f(t)$ is measurable in $[0, \infty)$,

( $\gamma) f(t)$ is continuous at a fixed point $x>0$,

$(\delta)$ there exist constants $m$ and $M$ such that $|f(t)| \leqslant M e^{m t^{2}}$ for every $t \geqslant 0$, then $P_{n}(f ; x) \rightarrow f(x)$ as $n \rightarrow \infty$.

COROLLARY 2. If

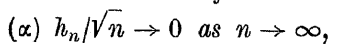

( $\beta) f(t)$ is continuous in the interval $[0, \infty)$,

$(\gamma)$ there exist constants $m$ and $M$ such that $|f(t)| \leqslant M e^{m t^{2}}$ for every $t \geqslant 0$, then the sequence $P_{n}(f ; x)$ is convergent to $f(x)$ almost uniformly in $(0, \infty)$.

Proof of corollary 2 (corollary 1 can be proved analogously). By theorem 3 it is sufficient to prove that sequence (2) is convergent to zero almost uniformly in $(0, \infty)$. Let $x \in[a, b]$, where $a>0$. We have $2 m b\left(h_{n} / \sqrt{n}\right) \leqslant 1$ and $m\left(h_{n} / \sqrt{n}\right)^{2} \leqslant \frac{1}{2}$ for sufficiently large $n$. Hence

$$
\begin{gathered}
\left|I_{n}(f ; x)\right|=\int_{\delta_{n} \sqrt{n} / h_{n}}^{\left(1-x / h_{n}\right) \sqrt{n}} f\left(x+\frac{h_{n}}{\sqrt{n}} u\right)\left(1-\frac{u^{2}}{n}\right)^{n} d u \mid \leqslant M \int_{\delta_{n} \sqrt{n} / h_{n}}^{\left(1-x / h_{n}\right) \sqrt{n}} e^{m\left(x+h_{n} u / \sqrt{n}\right)^{2}} e^{-u^{2}} d u \\
\leqslant M e^{m b^{2}} \int_{\delta_{n} \sqrt{n} / h_{n}}^{\left(1-a / h_{n}\right) \sqrt{n}} e^{u-\frac{1}{2} u^{2}} d u .
\end{gathered}
$$

The integral $\int_{0}^{\infty} e^{u-\frac{1}{2} u^{2}} d u$ being convergent, the sequence $I_{n}(f ; x)$ is convergent to zero uniformly in $[a, b]$, whence almost uniformly in $(0, \infty)$.
The following question arises: are the polynomials $P_{n}(f ; x)$ convergent for functions increasing more rapidly than $e^{-t^{2}}$ ? The answer depends on the choice of the sequence $h_{n}$. We consider the following example:

If the sequence $h_{n}$ satisfies the conditions $h_{n} / \sqrt{n} \rightarrow 0, \limsup _{n \rightarrow \infty} h_{n}^{s} / n>$ $>0$, where $s>2$, then $\limsup _{n \rightarrow \infty} P_{n}\left(e^{t^{s+\varepsilon}} ; x\right)=\infty$ for arbitrary $x, \varepsilon>0$.

By identity (3) it is sufficient to show $\limsup _{n \rightarrow \infty} I_{n}\left(e^{t^{8+\varepsilon}} ; x\right)=\infty$. Since $\delta_{n} / h_{n}<\frac{1}{2}\left(1-x / h_{n}\right)$ for sufficiently large $n$, we have

$$
\begin{aligned}
I_{n} & =\int_{\delta_{n} \sqrt{n} / h_{n}}^{\left(1-x / h_{n}\right) \sqrt{n}}\left(1-\frac{t^{2}}{n}\right)^{n} \exp \left(x+\frac{h_{n}}{\sqrt{n}} t\right)^{s+\varepsilon} d t \\
& \geqslant \int_{\frac{1}{2}\left(1-x / h_{n}\right) \sqrt{n}}^{\left(1-x / h_{n}\right) \sqrt{n}}\left(1-\frac{t^{2}}{n}\right)^{n} \exp \left(x+\frac{h_{n}}{\sqrt{n}} t\right)^{s+\varepsilon} d t \\
& \geqslant \int_{\left(1-x / h_{n}\right) \sqrt{n}}^{\frac{1}{2}\left(1-x / h_{n}\right) \sqrt{n}}\left[1-\left(1-\frac{x}{h_{n}}\right)^{2}\right]^{n} \exp \left[x+\frac{1}{2} h_{n}\left(1-\frac{x}{h_{n}}\right)\right]^{s+\varepsilon} d t \\
& =\frac{1}{2}\left(1-\frac{x}{h_{n}}\right) \sqrt{n} \exp \left\{n h_{n}^{\varepsilon}\left[\frac{h_{n}^{s}}{n}\left(\frac{x+h_{n}}{2 h_{n}}\right)^{s+\varepsilon}+\frac{\lg x-\lg h_{n}+\lg \left(2-\frac{x}{h_{n}}\right)}{h_{n}^{e}}\right]\right\} \\
& \geqslant \frac{1}{2}\left(1-\frac{x}{h_{n}}\right) \sqrt{n}
\end{aligned}
$$

for infinitely many $n$, and this proves the statement.

\section{THEOREM 4. If}

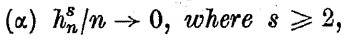

( $\beta) f(t)$ is measurable in $[0, \infty)$,

$(\gamma) f(t)$ is continuous at a fixed point $x>0$,

$(\delta)$ there exist constants $m$ and $M$ such that $|f(t)| \leqslant M e^{m t^{s}}$ for every $t \geqslant 0$, then $P_{n}(f ; x) \rightarrow f(x)$ as $n \rightarrow \infty$.

THEOREM 5. If

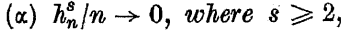

( $\beta$ ) $f(t)$. is continuous in the interval $[0, \infty)$,

$(\gamma)$ there exist constants $m$ and $M$ such that $|f(t)| \leqslant M e^{m t}$ for every $t \geqslant 0$, then the sequence $P_{n}(f ; x)$ is convergent to $f(x)$ almost uniformly in $(0, \infty)$. 
Proof of theorem 5 (theorem 4 can be proved similarly). By theorem 3 it is suffieient to show almost uniform convergence of sequence (2). Let $x \in[a, b]$, where $a>0$. We have

$$
\begin{aligned}
\left|I_{n}(f ; x)\right| & \leqslant M \int_{\delta_{n} \sqrt{n} / h_{n}}^{\left(1-x / h_{n}\right) \sqrt{n}} \exp \left[m\left(x+\frac{h_{n}}{\sqrt{n}} t\right)^{s}-t^{2}\right] d t \\
& \leqslant M \int_{\delta_{n} \sqrt{n} / h_{n}}^{\left(1-a / h_{n}\right) \sqrt{n}} \exp \left\{t^{2}\left[m\left(\frac{b}{\left(\delta_{n} \sqrt{n} / h_{n}\right)^{2 / s}}+\frac{h_{n}}{n^{1 / s}}\left(1-\frac{a}{h_{n}}\right)^{1-2 / s}\right)^{s}-1\right]\right\} d t \\
& \leqslant M \int_{\delta_{n} \sqrt{n} / h_{n}}^{\left(1-a / h_{n}\right) \sqrt{n}} \exp \left(-\frac{1}{2} t^{2}\right) d t
\end{aligned}
$$

for sufficiently large $n$, since the expression in brackets is less than $-\frac{1}{2}$. The interval $[a, b]$ being arbitrary, the sequence $I_{n}$ tends to zero almost uniformly in $(0, \infty)$.

THEOREM 6. If

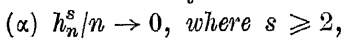

( $\beta) f(t)$ is measurable in $[0, \infty)$,

$(\gamma)$ the 27 -th $(k \geqslant 1)$ derivative $f^{(2 k)}(x)$ exists at a fixed point $x>0$,

$(\delta)$ there exist constants $m$ and $M$ such that $|f(t)| \leqslant M e^{m t^{s}}$ for every $t \geqslant 0$, then

$$
\lim _{n \rightarrow \infty}\left(\frac{\sqrt{n}}{h_{n}}\right)^{2 k}\left\{P_{n}[f(t) ; x]-\sum_{r=0}^{2 k-1} \frac{f^{(r)}(x)}{r !} P_{n}\left[(t-x)^{r} ; x\right]\right\}=\frac{\Gamma\left(k+\frac{1}{2}\right)}{\Gamma\left(\frac{1}{2}\right)} \frac{f^{(2 k)}(x)}{(2 k) !}
$$

Proof. By the assumption $(\gamma)$,

(5) $f(t)=f(x)+\frac{f^{\prime}(x)}{1 !}(t-x)+\ldots+\frac{f^{(2 k)}(x)}{(2 k) !}(t-x)^{2 k}+(t-x)^{2 k} \eta(t-x)$,

where $\lim _{u \rightarrow 0} \eta(u)=0$. Next, by the assumption $(\delta)$, there is a constant $L(x)$ such that $|\eta(u)| \leqslant L(x) \exp \left[m(x+u)^{s}\right]$ for every $u \geqslant-x$. Applying (5) we obtain

(6) $\quad\left(\frac{\sqrt{\bar{\eta}}}{h_{n}}\right)^{2 k}\left\{P_{n}[f(t) ; x]-\sum_{r=0}^{2 k-1} \frac{f^{(r)}(x)}{r !} P_{n}\left[(t-x)^{r} ; x\right]\right\}$

$$
=\frac{f^{(2 k)}(x)}{(2 k) !}\left(\frac{\sqrt{n}}{h_{n}}\right)^{2 k} P_{n}\left[(t-x)^{2 k} ; x\right]+\left(\frac{\sqrt{n}}{h_{n}}\right)^{2 k} P_{n}\left[(t-x)^{2 k} \eta(t-x) ; x\right] .
$$

Easy transformations yield

$$
\left(\frac{\sqrt{n}}{h_{n}}\right)^{2 k} P_{n}\left[(t-x)^{2 k} ; x\right]=n^{k} \frac{B\left(k+\frac{1}{2} ; n+1\right)}{B\left(\frac{1}{2} ; n+1\right)}-r_{n}(x),
$$

where

$$
r_{n}(x)=\frac{\int_{x \sqrt{n} / h_{n}}^{\sqrt{n}} t^{2 k}\left(1-t^{2} / n\right)^{n} d t+\int_{\left(1-x / h_{n}\right) \sqrt{n}}^{\sqrt{n}} t^{2 k}\left(1-t^{2} / n\right)^{n} d t}{2 \sqrt{n} \int_{0}^{1}\left(1-t^{2}\right)^{n} d t .} .
$$

However, we have for sufficiently large $n$

and

$$
0 \leqslant \int_{x \sqrt{n} / h_{n}}^{\sqrt{n}} u^{2 k}\left(1-\frac{u^{2}}{n}\right)^{n} d u \leqslant \int_{x \sqrt{n} / h_{n}}^{\sqrt{n}} u^{2 k} e^{-u^{2}} d u
$$

$$
0 \leqslant \int_{\left(1-x / h_{n}\right) \sqrt{n}}^{\sqrt{n}} u^{2 k}\left(1-\frac{u^{2}}{n}\right)^{n} d u \leqslant \int_{\left(1-x / h_{n}\right) \sqrt{n}}^{\sqrt{n}} u^{2 k} e^{-u^{2}} d u
$$

This proves that $r_{n}(x) \rightarrow 0$, for the integral $\int_{0}^{\infty} u^{2 k} e^{-u^{2}} d u$ is convergent. Applying the well-known relation between the functions $B$ and $\Gamma$ and the functional equation of the function $\Gamma$ we obtain

$$
\begin{aligned}
& n^{k} \frac{B\left(k+\frac{1}{2} ; n+1\right)}{B\left(\frac{1}{2} ; n+1\right)}=n^{k} \frac{\Gamma\left(k+\frac{1}{2}\right) \Gamma(n+1)}{\Gamma\left(n+1+k+\frac{1}{2}\right)} \frac{\Gamma\left(n+1+\frac{1}{2}\right)}{\Gamma\left(\frac{1}{2}\right) \Gamma(n+1)} \\
& =n^{k} \frac{\frac{1}{2}\left(\frac{1}{2}+1\right) \ldots\left(\frac{1}{2}+n\right)}{\left(k+\frac{1}{2}\right)\left(k+\frac{1}{2}+1\right) \ldots\left(k+\frac{1}{2}+n\right)} \\
& =\frac{n ! n^{k+\frac{1}{2}}}{\left(k+\frac{1}{2}\right)\left(k+\frac{1}{2}+1\right) \ldots\left(k+\frac{1}{2}+n\right)} \frac{\frac{1}{2}\left(\frac{1}{2}+1\right) \ldots\left(\frac{1}{2}+n\right)}{n ! n^{1 / 2}} \rightarrow \frac{\Gamma\left(k+\frac{1}{2}\right)}{\Gamma\left(\frac{1}{2}\right)}
\end{aligned}
$$

for $\Gamma(z)=\lim _{n \rightarrow \infty} \frac{n ! n^{z}}{z(z+1) \ldots(z+n)}$.

By (7) we get

$$
\lim _{n \rightarrow \infty}\left(\frac{\sqrt{n}}{h_{n}}\right)^{2 k} P_{n}\left[(t-x)^{2 k} ; x\right]=\frac{\Gamma\left(k+\frac{1}{2}\right)}{\Gamma\left(\frac{1}{2}\right)} .
$$

Let $\delta_{n}$ satisfy the conditions $\delta_{n}>0, \delta_{n} \rightarrow 0, \delta_{n} \sqrt{n} / h_{n} \rightarrow \infty$. We have 


$$
\begin{aligned}
& \left|\left(\frac{\sqrt{n}}{h_{n}}\right)^{2 k} P_{n}\left[(t-x)^{2 k} \eta(t-x) ; x\right]\right| \\
\leqslant & \left(\frac{\sqrt{n}}{h_{n}}\right)^{2 k} \frac{\left(\int_{x-\delta_{n}}^{x+\delta_{n}}+\int_{0}^{x-\delta_{n}}+\int_{x+\delta_{n}}^{n_{n}}\right)(t-x)^{2 k}|\eta(t-x)|\left[1-\left(\frac{t-x}{h_{n}}\right)^{2}\right]^{n} d t}{2 h_{n} \int_{0}^{1}\left(1-t^{2}\right)^{n} d t}
\end{aligned}
$$$$
\leqslant \frac{\sup _{|u| \leqslant \delta_{n}}|\eta(u)| \int_{-\delta_{n} \sqrt{n} / h n}^{\delta_{n} \sqrt{n} / h n} u^{2 k} e^{-u^{2}} d u}{2 \sqrt{n} \int_{0}^{1}\left(1-t^{2}\right)^{n} d t}+
$$

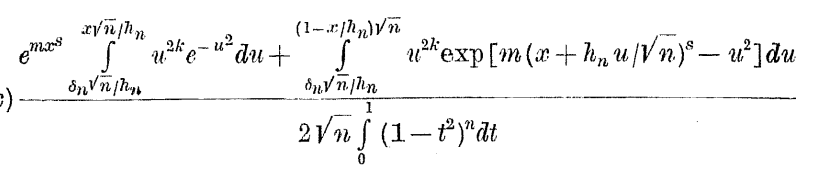$$
\leqslant \sup _{|u| \leqslant \delta, ~}|\eta(u)| \int_{-\infty}^{+\infty} u^{2 k} e^{-u^{2}} d u+L(x)\left\{e^{m x^{s}} \int_{\delta_{n} / \bar{n} / h_{n}}^{x_{2} / \bar{n} / h_{n}} u^{2 k} e^{-u^{2}} d u+\right.
$$

$$
+\int_{\delta_{n} / n / h_{n}}^{\left(1-x / h_{n}\right) \sqrt{n}} u^{2 k} \exp \left\{u^{2}\left[m\left(\frac{x}{\left(\delta_{n} \sqrt{n} / h_{n}\right)^{2 / s}}+\frac{h_{n}}{n^{1 / s}}\left(1-\frac{x}{h_{n}}\right)^{1-2 / s}\right)^{s}-1\right]\right\} d u
$$

for sufficiently large $n$. Since $\lim \eta(u)=0$ and the expression in brackets is less than $-\frac{1}{2}$ for sufficiently large $n$, we have

$$
\lim _{n \rightarrow \infty}\left(\frac{\sqrt{n}}{h_{n}}\right)^{2 k} P_{n}\left[(t-x)^{2 k} \eta(t-x) ; x\right]=0 .
$$

Applying (8) and (9) we obtain the theorem from (6).

\section{References}

[1] L.C. Hsu, The polynomial approximation of continuous functions defined on $(-\infty, \infty)$, Czechoslovak Mat. Journal 9 (1959), p. 574-578.

[2] - Approximation of non-bounded continuous functions by certain sequences of linear positive operators or polynomials, Studia Mathematica 21 (1961), p. 37-43.

Reçu par la Rédaction le 26.6.1961

\section{Entire functions in $B_{0}$-algebras}

B. MITIAGIN (Moscow), S. ROLEWICZ and W. ZELAZEO (Warszawa)

A $B_{0}$-algebra is a completely metrizable locally convex topological algebra over the real or complex scalars. We shall also assume that the algebras in question possess unit elements.

The topology in a $B_{0}$-algebra $R$ may be introduced by means of a denumerable sequence of pseudonorms satisfying

and

$$
\|x\|_{i} \leqslant\|x\|_{i+1}, \quad i=1,2, \ldots,
$$

(see [13], theorem 24). A sequence $x_{n}$ tends to $x_{0}$ if and only if $\lim \left\|x_{n}-x_{0}\right\|_{i}$ $=0, i=1,2, \ldots$ The basis of neighbourhoods of zero in $R$ is of the form $\left\{K_{i}(1 / n)\right\} \quad(i, n=1,2, \ldots)$, where $K_{i}(r)=\left\{x \in R:\|x\|_{i}<r\right\}$. Any subsequence of the sequence $\left\{\|x\|_{i}\right\}$ also satisfies (1) and (2) and gives in $R$ the same topology.

A $B_{0}$-algebra $R$ is called m-convex if there exists an equivalent system of pseudonorms satisfying

$$
\|x y\|_{i} \leqslant\|x\|_{i}\|y\|_{i}, \quad i=1,2, \ldots
$$

The concept of an $m$-convex $B_{0}$-algebra, first introduced by Arens [2], was then considered in detail by Michael in [7]. A $B_{0}$-algebra is $m$ -convex if and only if there exists a fundamental system $\{U\}$ of neighbourhoods of 0 which are idempodent (i. e. such that $U U \subset U$, where $X Y=\{z \in R: z=x y, x \in X, y \in Y\}, X, Y$ - arbitrary subsets of $R$ ), or if there exists an equivalent system of pseudonorms such that multiplication is continuous with respect to each one [7]. In [7] it is also shown that if $U$ is an idempotent subset of $R$, then so are its convex hull conv $U$ and its closure $\bar{U}$.

If $R$ is an $m$-convex $B_{0}$-algebra and $\varphi(z)=\sum_{n=0}^{\infty} a_{n} z^{n}$ is an entire function of complex variable $z$, then for every $x \in R$ the series $\varphi(x)=\sum_{0}^{\infty} a_{n} x^{n}$ 